\title{
EL ESPEJISMO \\ DE LA EFICIENCIA ECONÓMICA
}

\author{
IGNACIO DE LEÓN*
}

Resumen: Existe un consenso internacional creciente acerca de la necesidad de impulsar la eficiencia económica como principio rector de la formulación de políticas públicas en el campo económico, especialmente para promover la transición de las economías de países en desarrollo hacia los mercados. Este ensayo objeta las premisas intelectuales que sustentan esa creencia, y expone por qué la eficiencia económica representa un criterio normativo de asignación de los recursos sociales que obstaculiza el proceso creador de los mercados. Dadas las condiciones utópicas bajo las cuales se construye dicho cálculo de coste-beneficio de bienestar social, no es posible su aplicación normativa para juzgar la conducta de los empresarios en los mercados. La eficiencia económica deja de lado todo contacto con la dinámica evolutiva, renovadora y creadora de los mercados. Por ello, distrae la atención del regulador hacia problemas irrelevantes para la promoción del libre mercado. Empleando las ideas de proceso de mercado de F.A. Hayek, I. Kirzner y G.B.Richardson, este ensayo muestra que el desarrollo de inversiones en los mercados, base del crecimiento económico, está ligado a que los empresarios obtengan la información útil que necesitan para hacer inversiones. Se muestra de qué manera las políticas de competencia, al fundarse en una visión epistemológica estructural y estática de los mercados, terminan por distorsionar su propia finalidad de políticas públicas.

Palabras clave: Eficiencia económica, políticas públicas.

Abstract: There is an international growing consensus about the need of promoting economic efficiency as the yardstick of modern economic policymaking, especially in the promotion of market transition in developing countries. This essay objects the supporting premises of such assumption. It exposes why economic efficiency represents a normative criterion for the assignment of social resources which undermines markets' creative functioning. Given the utopian founding premises within which such

(*) Abogado, LL.M., M. Phil., Ph.D. Ex Superintendente de la Superintendencia para la Promoción y Protección de la Libre Competencia de Venezuela. 
social welfare cost-benefit analysis is built, its enforcement cannot be meaningfully applied to judge entrepreneurs' market conduct. Economic efficiency leaves aside all connection with the creative evolutionary dynamics of markets. Because of this, it diverts the attention of regulatory authorities into policy problems which are irrelevant, even counterproductive, for the promotion of free markets. By applying market process ideas expounded by F.A. Hayek, I. Kirzner and G.B.Richardson, this essay shows that investment promotion, which is the very foundation of economic growth, is linked to entrepreneurs' seizure of useful information which they need to undertake their investments. This essay shows how competition policies, by grounding their views in a structural and static vision of markets, end up distorting their very policy purpose.

Key words: Economic efficiency, public policies.

Clasificación JEL: B53, D61, D83, D84

I.

INTRODUCCIÓN

Este ensayo explica el papel normativo desempeñado por la «eficiencia económica» en la aplicación de las políticas de competencia, así como sus limitaciones. Se pretende con ello, arrojar luces sobre el papel que desempeñan los valores, la ideología e instituciones en la regulación económica, tal como es aplicada modernamente, y los desafíos prácticos que presenta el diseño de políticas públicas para la adopción de políticas orientadas a la promoción del mercado como estrategia central del desarrollo económico.

Existe un consenso creciente acerca de la necesidad de incorporar la eficiencia económica y la competencia como principios rectores de la formulación de políticas públicas en el campo económico, a todo nivel. ${ }^{1}$ Esto supone el triunfo nominal

1 Véase Crampton (2004). 
de aquellos que defienden la asignación de los mercados sobre la planificación estatal como motor del desarrollo económico.

Así, en comparación con las viejas políticas de distribución para el desarrollo aplicadas en América Latina, la asignación «eficiente» pone los recursos sociales en las manos de aquellos que más los valoran. Esto asegura el funcionamiento óptimo del mercado, siempre y cuando no medien costes de transacción. De acuerdo con esta perspectiva, la eficiencia económica proporciona una referencia normativa «objetiva e imparcial» desde la cual es posible proponer asignaciones de recursos que incrementan el bienestar social sin riesgo de interferirlas por consideraciones distributivas que, por su indefinición concreta, tienden a elevar la discrecionalidad gubernamental en aras de alcanzar una nebulosa equidad. Esta suele ser la visión que prevalece en la actualidad en los programas de reforma estructural practicados en los países en desarrollo.

Este ensayo cuestiona esta visión autocomplaciente, al afirmar que el estándar convencional de eficiencia económica posee deficiencias epistemológicas graves que anulan su valor como punto de referencia para la implementación de las políticas públicas. Este es el caso, particularmente, de las políticas de competencia, donde la aplicación del criterio de eficiencia económica puede incluso frustrar la dinámica competitiva de los mercados.

La eficiencia económica normativa (y su concepto correlativo, el modelo de competencia perfecta) tiende a desempeñar en el análisis de los mercados un papel semejante al de la ideología en los asuntos sociales, a saber, presenta al analista con un estándar normativo utópico que es imposible satisfacer; por lo que todas las transacciones aparecen como casos graves o leves de «imperfección de mercado» que deben ser intervenidos. Un estándar así concebido no proporciona la información que el regulador necesita para construir instituciones 
estables de mercado; por el contrario, lo invita intervenir en todo momento. Se trata, por lo tanto, de un estándar normativo que carece de toda utilidad práctica para ser referencia en la aplicación de políticas públicas, dado que su concepción es, desde una perspectiva epistemológica, insostenible.

Por esta razón, el espejismo de la eficiencia económica constituye un hábito de pensamiento en el investigador económico (independientemente de su posición en el caso que ha de juzgar: analista técnico, comisionado-autoridad de competencia, abogado litigante, empresa investigada), hacia problemas que nada tienen que ver con la construcción de instituciones que permitan fortalecer los mercados, que es lo fundamental para los países en desarrollo, y la razón de ser - si es que existe- que está detrás de la introducción de políticas de competencia.

La idea de que la visualización de las políticas públicas sobre los mercados está condicionada por los hábitos de pensamiento fue propuesta por F. A. Hayek: «Me parece que muchas de las controversias actuales sobre teoría económica y política pública tienen en común un malentendido sobre la naturaleza del problema económico de la sociedad. A su vez, este malentendido se debe a una aplicación errada sobre los fenómenos sociales de los hábitos de pensamiento que hemos desarrollado en el análisis de los fenómenos de la naturaleza.»² Estos «hábitos de pensamiento» han sido un factor decisivo de la percepción predominante entre los economistas sobre los fenómenos sociales, lo que vino a influenciar la dirección que habrían de tener las políticas públicas destinadas a fortalecer los mercados, especialmente a partir de la década de 1980, con la apertura económica producida en el mundo.

En Latinoamérica esta disyuntiva igualmente se materializó cuando se abandonaron las antiguas políticas «desarrollis-

2 Hayek (1948), p. 78. 
tas» y fueron reemplazadas por una estrategia más conforme al mercado. En ese momento adquirió sentido adoptar una legislación y unas políticas de competencia dirigidas a estimular la apertura de los mercados. Desde luego, siguiendo a Hayek, el sentido de estas políticas vino a estar dado, más allá de la retórica a favor de los mercados, por el entendimiento o comprensión de éstos en la mente de los encargados de diseñar las políticas de apertura económica. En última instancia, este vino a ser el factor determinante en la concepción de la nueva estrategia de crecimiento.

Por ello, conviene indagar con un poco más de profundidad en la esencia de las ideas acerca de la competencia y los mercados, según la visión predominante en el momento de producirse la apertura económica en Latinoamérica.

II.

EL ENFOQUE TRADICIONAL DE LAS POLÍTICAS DE COMPETENCIA: EL ROL NORMATIVO DEL MODELO DE COMPETENCIA PERFECTA (O EFECTIVA)

Para entonces, la agenda tradicional de las políticas de competencia había estado orientada a la búsqueda del Bienestar del Consumidor, procurando la asignación óptima eficiente de los recursos sociales escasos. Este enfoque de política de competencia, asociado con la Escuela de Chicago, ${ }^{3}$ fue concebido a lo largo de un proceso que se inició con la evolución jurisprudencial por los tribunales estadounidenses en aplicación de la Ley Sherman (1890) y otras sucesivas leyes pertenecientes al régimen antitrust, proceso éste que se vio complementado por los avances en la teoría económica de los mercados,

\footnotetext{
3 Bork (1978).
} 
especialmente en los trabajos de Chamberlin y Robinson hacia la década de 1930, sobre la competencia monopolística, trabajos éstos que derivaron hacia evaluaciones empíricas sobre las condiciones «imperfectas» de los mercados, y eventualmente, la importancia de las barreras a la entrada en la determinación de condiciones de competencia.

La visión convencional inicialmente dio lugar a una serie de intervenciones «estructurales» en el mercado dirigidas a atenuar lo que era percibido como una indebida concentración de poder económico, como se desprende de casos emblemáticos tales como Alcoa, Brown Shoe, y otros. ${ }^{4}$ Esta visión asociada con la llamada Escuela de Harvard, fue posteriormente abandonada, al surgir evidencia empírica que claramente relacionaba la concentración con otras explicaciones distintas de la búsqueda de ganancias monopólicas, tales como la realización de eficiencias económicas superiores. La explicación inicial según la cual la causalidad entre estructura y conducta es unidireccional fue reemplazada posteriormente por visiones «dinámicas» del problema, las cuales consideraban la concentración como resultado de estrategias corporativas ${ }^{5}$ sin otra finalidad que la generación de eficiencias económicas. ${ }^{6}$

Incluso, bajo la teoría de los «mercados contestables», lo esencial no es tanto la concentración como la existencia de barreras al ingreso o a la salida. ${ }^{7}$ A partir de estas ideas, se ha ido consolidando un consenso en la aplicación de las políticas de competencia aplicadas internacionalmente. Según este consenso, una política de competencia debe procurar esencialmente disciplinar las empresas participantes en el mercado, cuyo comportamiento habría de corregir «fallos

\footnotetext{
${ }^{4}$ Bork, op. cit.

Caves y Porter (1977).

6 Demsetz (1982), pp. 386-393.

7 Baumol, Panzar y Willig (1982).
} 
de mercado» cuya aparición haría caer los mercados en equilibrios sub-óptimos, y, por tanto, normativamente indeseables.

Por tanto, bajo esta visión estructural y estática asociada con la competencia como fenómeno de mercado se plantea una relación entre el comportamiento de las empresas con la estructura más o menos concentrada de los mercados.

Esta visión ha quedado recogida en el llamado «Paradigma S-C-P» (Structure- Conduct-Performance). Mientras más concentrados, en principio, cabe esperar que las prácticas restrictivas de la rivalidad en ellos sea la expresión de una intención monopolística por extraer rentas de un consumidor inerme.

Elementos tales como las barreras que hacen inviable la presencia de innumerables competidores en el mercado alejan los mercados «reales» de la competencia perfecta. La intervención del Estado es necesaria entonces para «acercar» la realidad «imperfecta» a una situación de mercado más «cercana» al equilibrio, y por tanto, a la asignación eficiente de los recursos.

He aquí el punto de partida del problema aquí examinado. Los defensores del criterio de eficiencia económica y el Bienestar del Consumidor como objetivos normativos de las políticas públicas de competencia presuponen que los mercados habrán de funcionar de modo óptimo cuando se comporten de modo semejante a los postulados del modelo de Competencia Perfecta. Como señalan Dorfman, Samuelson y Solow: «La perspectiva teórica estandar de la política económica se ha basado en el concepto de eficiencia de Pareto, y de modo igualmente fundamental, en la afirmación de que 'todo equilibrio competitivo es un óptimo de Pareto; y todo óptimo de Pareto es un equilibrio competitivo.» ${ }^{8}$ Una consideración similar es

\footnotetext{
8 Dorfman, Samuelson y Solow (1958), p. 410.
} 
aplicable al modelo de Competencia Efectiva, que, dadas las exigencias de sus supuestos, es simplemente un relajamiento de aquél, a fin de «acercarlo a la realidad.»

Klein enfatiza la importancia del modelo de competencia perfecta en el análisis antimonopolio, de la siguiente manera: «... de todas las herramientas analíticas que comprende la economía política contemporánea, quizás el más importante para el economista es el modelo de Competencia Perfecta.» ${ }^{9}$ De igual manera, Clark argumenta: «El concepto de 'competencia perfecta' ha recibido por primera vez una definición y elaboración especificas. Con ello ha devenido la convicción de que la 'competencia perfecta' no puede existir y presumiblemente nunca ha existido (...) Lo que ha quedado es un estándar irreal o ideal que puede servir de punto de partida para el análisis y una norma con la cual comparar las condiciones competitivas de la realidad. También ha servido como un estándar por el cual éstas se pueden juzgar.» ${ }^{10}$

Finalmente, en relación con este modelo, Hayek observó: «Este caso ideal (...) vino a ser considerado como el modelo, y fue empleado como el estándar por el cual se habría de juzgar el desempeño de la competencia en el mundo real.» ${ }^{11}$ Es claro entonces que nuestro objeto de análisis es el Modelo de Competencia Perfecta, en tanto representa el estándar normativo esencial de la política de competencia con el cual se evalúan las transacciones de mercado. Las consideraciones de este análisis son igualmente extensibles al modelo de Competencia Efectiva, por las mismas razones epistemológicas que inmediatamente se examinarán.

La visión tradicional no toma en cuenta los costes de información en que ha de incurrir la autoridad estatal para lograr

\footnotetext{
9 Klein (1990), p. 420.

10 Clark (1940), p. 241.

${ }^{11}$ Hayek (1976), p. 66. Un análisis reciente sobre el uso de la competencia perfecta como estándar normativo se encuentra en Burton (1994).
} 
el equilibrio óptimo de los mercados. Tampoco considera la crítica epistemológica, más profunda, que cuestiona el intento de comparar conductas «reales» de mercado con situaciones «ideales» expresadas en modelos.

El interés de la eficiencia económica como concepto normativo está relacionado en última instancia con la consecución de estados de bienestar social a través de la intervención pública. Esta pretensión descansa en la idea de que el investigador puede tener una perspectiva cabal de toda la complejidad de factores que caracteriza la realidad social, y manipular estos factores de tal manera que sea posible alcanzar el Bienestar Social. Sin embargo, a fin de alcanzar este objetivo, los analistas deben cumplir con dos condiciones.

En primer lugar, deben poseer las herramientas analíticas adecuadas para entender y valorar la realidad correctamente; a tal efecto emplean «modelos» de mercado que les permiten capturar la esencia de las fuerzas de mercado. En consecuencia es necesario explorar más en detalle la naturaleza de estos modelos, lo que realmente ellos representan en el análisis, y por supuesto, cuáles son sus grietas epistemológicas, a fin de poder comprender las limitaciones que contiene el análisis convencional de los mercados, aplicado en las políticas de competencia.

En segundo lugar, para alcanzar el Bienestar Social, los analistas deben estar en capacidad de identificar la «condición óptima» de los mercados; esto es, los costes que el sistema social (gobierno y empresas) debe soportar a fin de alcanzar este punto ideal. Es dudoso que una situación pueda calificarse de "optima» si el coste para hacerla realidad es superior a los beneficios que resultan de ella. No obstante, el investigador suele asumir que éste es un ejercicio sin coste alguno; esta creencia nace de la suposición de que el objetivo de la política pública es construir un mundo sin costes. 
Estas dos suposiciones están sólidamente ancladas en la mente de los investigadores económicos. Se asume la visión cartesiana de que la realidad es una entidad objetiva, localizada fuera de la mente, que puede ser completamente aprehendida y entendida; ${ }^{12}$ lo cual conduce a los responsables de las políticas públicas a un ejercicio dominado por lo que Hayek calificara como «Pretensión de Conocimiento», ${ }^{13}$ donde la «condición óptima», el «Bienestar Social», y otros sinónimos alusivos a la Utilidad Social aparecen como accesibles, convirtiéndose de este modo en un imperativo moral que los responsables de las políticas públicas están obligados a hacer realidad.

III.

LA PRIMERA CRÍTICA:

\section{LA FALACIA DE NIRVANA}

La visión de Nirvana es semejante a la idea de la «Justicia Perfecta» que propone Richard Epstein. ${ }^{14}$ Para ser alcanzada, la Justicia Perfecta requiere eliminar el error en cada caso individual, independientemente de los costes en que haya que incurrir para hacer esto posible. ${ }^{15}$ Del mismo modo, Sowell refiere el concepto de «Justicia Cósmica», esto es una justicia que es gratuita, sin coste, y que calibra la posición de bienestar individual de cada sujeto en la sociedad, a fin de igualar

12 Hayek (1973). También, de una manera brillante, Karl Popper contradijo la creencia de que la mente es una «caja» que recibe información o «data» del mundo exterior a través de los sentidos. En realidad, el «conocimiento» es construido a través de la interpretación continua que el observador hace de la realidad que le rodea, de acuerdo con su entendimiento (y valores) y percepción auto creada. Véase Popper (1990).

13 Conferencia magistral con ocasión de recibir el Premio Nobel de Economía en 1974.

14 Epstein (1995).

15 Epstein (1995), p. 38. 
su condición a la del resto de los individuos. Sowell critica este ejercicio, sobre la base de que es imposible visualizar un estándar ideal de igualdad que pueda satisfacer la condición de cada individuo por igual, dado el coste que semejante ejercicio supone. Así, "con la justicia, al igual que con igualdad, la pregunta no es si más de ella es mejor, sino si es mejor a cualquier costo». ${ }^{16}$

En la visión de Sowell, «aquellos que pretenden alcanzar la Justicia Cósmica tienden a suponer que tendría como consecuencia aquello que ellos desean; esto es, que las personas sujetas a las políticas gubernamentales serían como piezas en un tablero de ajedrez que podrían ser movidas de un sitio para otro, a fin de ejecutar un gran diseño social, sin consideración alguna a las propias reacciones.» 17

En el campo de la ciencia económica predomina una forma de pensamiento semejante, esto es, se da por sentado que la Autoridad puede alcanzar el Bienestar Social a partir de las manipulaciones que introduce en el comportamiento de los agentes económicos que se «desvían» del ideal de competencia perfecta. El modelo de Competencia Efectiva aplica la misma lógica, a saber, que en nuestras mentes podemos concebir situaciones que nos permiten apreciar cómo las cosas podrían ser diferentes si no viviéramos en un mundo lleno de fallos de mercado.

Sin embargo, el coste que es necesario asumir para alcanzar un estado «deseable», es inevitable en cualquier ejercicio de política pública (o de la vida). Por ello, quizás convenga distinguir lo «deseable» de lo «utópico», y aclarar que la condición «ideal» de los modelos es una simple referencia al anclaje de los mismos en las «ideas», y no a su condición de referencia normativa, u objetivo «deseable» a alcanzar. Los estándares ideales-utópicos

\footnotetext{
16 Sowell (1999), p. 27.

17 Id. at 40 .
} 
dejan fuera del análisis el coste de adquirir la información que se requiere para poder alcanzar la condición deseada. Éste es un coste social, tanto para las empresas como para la autoridad gubernamental. Contrastar la realidad con la condición utópica del Nirvana no toma en cuenta los costes en que los miembros de la sociedad deben incurrir en a fin de llevar adelante sus inversiones y decisiones productivas, como veremos enseguida. Estas decisiones de inversión (o de abstenerse de invertir) nunca tendrían lugar, y por tanto, no podrían ser consideradas como parte del análisis regulatorio, in abstracto, sino que sólo pueden producirse una vez que los inversionistas han internalizado sus costes.

Demsetz se refirió a esta manera de pensar como la «Falacia del Nirvana», es decir, el error intelectual de considerar que podemos alcanzar un mundo de perfección dejando de lado lo difícil que para el investigador, la autoridad pública, etc., es conseguir la información necesaria para que la condición deseable efectivamente acontezca, e igualmente, ignorando los costes que han de soportar los empresarios a fin de poder hacer la información accesible; información que precisamente requieren para poder llevar el sistema, de una situación de desequilibrio, a una condición de equilibrio eficiente.

Evidentemente, la inclinación de los que caen en esta trampa intelectual es, como gráficamente afirmaba Demsetz, «creer que el jardín del vecino siempre es más bonito que el de uno». Así, comparado con el Nirvana, la realidad siempre parece estar plagada de «fallos de mercado». ${ }^{18}$

Éste es un hecho del mundo real que los reguladores simplemente no pueden ignorar. Considérese el siguiente ejemplo. Imaginemos, que visitamos un gimnasio infantil y le preguntamos a los niños si están dispuestos a hacer los sacrificios

18 Tal como fuera expuesto por Demsetz (1969), «the neighbor's garden always looks greener». 
necesarios para convertirse en campeones olímpicos el día de mañana. Posiblemente obtengamos muchas respuestas positivas, a pesar de que en realidad quizás solamente uno de cada 10.000 gimnastas esté dispuesto a pagar el precio que se requiere para convertirse en un campeón olímpico. El análisis de eficiencia supone que siempre estaríamos mejor si solamente preguntáramos a los gimnastas si ellos están dispuestos a sacrificarse para llegar hasta las Olimpiadas, y después designar aquellos que han sido más enfáticos en manifestar su deseo para llegar al equipo olímpico.

En opinión de aquellos que promueven esta forma de pensar, este ejercicio intelectual ciertamente ahorraría todo el tiempo que requiere hacer un costoso entrenamiento. Por su parte, la realidad «falla» porque hay muchos gimnastas que gastarían tiempo de entrenamiento a pesar de que solamente unos pocos tendrían la posibilidad de llegar a las Olimpiadas; muchos quedarán en el camino, y esto representa en la visión convencional de eficiencia un coste social inútil.

IV.

LA SEGUNDA CRÍTICA:

LA INCONSISTENCIA LÓGICA DEL MODELO DE COMPETENCIA PERFECTA PARA APORTAR CRITERIOS NORMATIVOS SOBRE EL MERCADO

En el mundo de los negocios, la comparación entre un ideal de perfección y el mundo de los negocios deja al regulador con la impresión permanente de que cualquier conducta en los negocios que suponga un mínimo grado de cooperación es sospechosa de subvertir la competencia (perfecta o efectiva), puesto que los empresarios incurren en costes limitando sus propias posibilidades de acción, esto es, limitan su rivalidad, cooperan, a fin de alcanzar cierto grado de certidumbre sobre qué tipo de 
inversiones productivas pueden o no realizar. Desde la perspectiva de la eficiencia económica, estas limitaciones en la rivalidad siempre son vistas como interferencias, pues representan un alejamiento de los presupuestos del Modelo de Competencia Perfecta.

Esta visión no examina si dicha limitaciones son de hecho necesarias para que los empresarios puedan hacer realidad una oportunidad de negocio que, para acontecer, debe necesariamente desplazar a otros competidores de mercado.

El propósito de la competencia supone el éxito de unos empresarios alertas para percibir una oportunidad antes que los demás; esto no significa necesariamente que tales empresarios hayan obtenido esas oportunidades a expensas de otros. Solamente es posible en un escenario que supone la capacidad por igual de los otros para descubrir y aprovechar tales oportunidades; pero sabemos que esto es imposible, pues el sentido común nos dice que una oportunidad de negocios igualmente disponible para todos no lo está para nadie.

El Modelo de Competencia Perfecta no puede explicar cómo a partir de una situación de desequilibrio es posible llegar al equilibrio óptimo; peor aún, es imposible explicar cómo el sistema, estando en desequilibrio, puede moverse siquiera a otra posición menos desequilibrada. Para ello los participantes del sistema necesitan una información que sólo puede ser provista con en un entorno institucional y de «organización económica» (como la llama Richardson) que los modelos neoclásicos «postulan», pero no «explican». Pero he aquí que al postular la información completa, es imposible explicar cómo pueden moverse los agentes cuya situación es desequilibrada. La contradicción del objetivo (alcanzar el equilibrio óptimo) con los presupuestos necesarios para alcanzarlo (información completa) queda bien ilustrada en el ejemplo que aporta Oskar Morgerstern, quien demostró que, cuando el plan de un individuo es dependiente del plan de otro, el conocimiento perfecto 
produciría una cadena infinita de reacciones y contra-reacciones en las conjeturas de cada cual, lo que haría imposible el equilibrio.

Para ilustrar el punto, propuso como ejemplo la persecución entre Holmes y Moriarty: «Sherlock Holmes, perseguido por su oponente Moriarty, abandona Londres hacia Dover. El tren se detiene en una estación intermedia, y él resuelve apearse antes que continuar hacia Dover. Había visto a Moriarty en la estación Victoria de Londres, y conociendo la sagacidad de éste, esperaba que tomara un tren más rápido para llegar antes que él a Dover. La sospecha de Holmes era, en efecto, correcta. Pero, ¿Qué tal si Moriarty, siendo aún más suspicaz de lo que suponía Holmes, había evaluado correctamente la capacidad de éste y había anticipado su acción? En tal caso, hubiera decidido ir directamente a la estación intermedia, a esperarlo. Entonces, Holmes, nuevamente, hubiera anticipado tal acción y habría decidido seguir hasta Dover, en lugar de bajarse del tren. Con lo cual Moriarty hubiese de nuevo "reaccionado" de manera diferente. Después de pensarlo mucho, es probable que no hubiesen podido actuar en absoluto, o que aquel intelectualmente inferior se hubiese rendido en la estación Victoria de Londres, ya que todo el viaje hubiese sido innecesario.»19 La moraleja de esta historia es que la información perfecta de cada uno sobre los planes del otro hace que sea imposible que el sistema «se mueva» para alcanzar el nuevo equilibrio, ya que el movimiento (vale decir, la competencia) es por definición excluido del sistema. Por lo tanto, suponer que unos empresarios desplazan a otros porque aprovechan una oportunidad primero, es un error epistemológico que no toma en cuenta la forzada necesidad de que sólo sean algunos los que puedan aprovecharse de tal oportunidad, a fin de que el sistema pueda «moverse» y alguien salga beneficiado con ello.

19 O. Morgerstern, citado por O’Driscoll y Rizzo (1985), p. 84. 
Claramente se observa que, aplicar el Modelo de Competencia Perfecta (o Efectiva) como una referencia normativa puede llevar a una contradicción en cuanto los fines que persigue la política. En otras palabras, en vez de promover la capacidad de apercibimiento empresarial sobre las oportunidades de negocios a que da origen la competencia, induce a los reguladores a suponer que la competencia es menos probable mientras más nos alejemos de la situación «ideal».

Esta crítica ha sido propuesta por G.B. Richardson, en Information and Investment, ${ }^{20}$ al cuestionar la consistencia lógica misma del Modelo de Competencia Perfecta, que es utilizado ordinariamente como referencia normativa. Este es un ataque a la línea de flote de todo el sistema conceptual sobre el cual se asienta la visión convencional de los «fallos de mercado». ¿Cómo puede haber «fallos» si la referencia conceptual empleada para identificarla (el modelo de Competencia Perfecta) es inconsistente con su propósito normativo? La situación sería comparable a la de un barco que navega en las tinieblas, utilizando una brújula desmagnetizada.

Según Richardson, tales modelos ideales carecen de sentido como referencia normativa, por la sencilla razón de que estos modelos prescinden del entorno institucional que los agentes económicos requieren conocer para poder competir con otros empresarios y estructurar sus negocios. Así, la información que los agentes económicos necesitan para poder competir en los mercados y de ese modo llevar, en el largo plazo, los mercados a una situación de equilibrio, solamente es posible si se cuenta con la organización económica e institucional necesaria para hacerla efectiva. Por lo tanto aquellos modelos que no incorporan la estructura institucional necesaria como parte de su análisis (y éste es el caso de los modelos de competencia perfecta y de competencia efectiva), necesariamente

20 Richardson (1960). 
interpretan una causalidad de hechos que solamente se dan en un mundo ficticio, un mundo irreal.

Así, en Information and Investment Richardson destacaba la importancia de la calidad de la información de los participantes de mercado, a fin de que estos tomen decisiones de inversión. El primer capítulo de dicho libro lo dedicaba Richardson a criticar la insuficiencia del Modelo de Competencia Perfecta, y del enfoque económico tradicional en general, para dar respuesta a la pregunta esencial que debería hacerse el economista, a saber, ¿cómo obtienen los agentes económicos la información que necesitan para tomar sus decisiones de inversión? Naturalmente, el modelo de Competencia Perfecta, utilizado como base del análisis, es absolutamente inútil, en opinión de Richardson, pues para empezar, de partida presupone que la información que se necesita para alcanzar la condición de equilibrio propia de los sistemas capitalistas donde los recursos son asignados por decisiones de agentes económicos independientes y no por la planificación central, está plenamente disponible, pues esos son los postulados del modelo. En otras palabras, el modelo no resuelve la duda relativa a de dónde proviene la información.

Así, la crítica de Richardson no tiene nada que ver con el realismo de los supuestos empleados. Ciertamente, esta crítica ya había sido objetada por Friedman, quien había señalado que la utilidad de los modelos económicos radica no en adecuar sus premisas a la realidad, sino en su capacidad de predicción. ${ }^{21}$ Pero es que la crítica de Richardson va más allá del problema de los supuestos «reales» de los modelos. Tiene que ver con el hecho palmario de que el modelo es intrínsecamente inconsistente con su propio propósito normativo y en su propio postulado, pues, ¿cómo puede alcanzarse un equilibrio si no es a través del supuesto de que los agentes disponen de una información

21 Friedman (1953). 
a la que les es imposible acceder? Por ello las mismas suposiciones que de otro modo harían el modelo útil a los fines de diseñar políticas públicas esto es, que la información en el sistema así modelado efectivamente se transmite a todos los agentes económicos, haciendo posible el equilibrio y permitiendo los mercados competitivos, son condiciones no sólo inexistentes en el mundo real, sino más importante aún, son condiciones negadas por el modelo mismo. Esto es así, dado que no hay ninguna otra posibilidad de explicar de qué manera los sistemas sociales en desequilibrio llegan al equilibrio salvo suponiendo que la información en el sistema ya es conocida por los agentes económicos antes de que ella se haya transmitido. Pura esquizofrenia.

Así, estando en el equilibrio, el modelo asume que la información ya ha sido transmitida a los individuos que entonces descansarán en sus acciones; sin embargo, a fin de que esto suceda, los individuos deben coordinar sus acciones, construir rutinas y crear reglas que permitan la codificación y transmisión de la información a fin de que pueda ser entendida por todos en el sistema. De esta manera, y contrariamente a lo que asume el modelo, la coordinación, la creación de reglas y la estandarización del comportamiento de mercado que posibilita la transmisión de información, no acerca, sino que aleja los mercados reales del punto óptimo predicado por el modelo de competencia perfecta.

Es paradójico que, a fin de que el sistema alcance el equilibrio (y los mercados, la desconcentración cabal), los individuos se vean obligados a coordinar sus acciones (o a excluir a otros competidores unilateralmente, aprovechando primero la oportunidad), pero he aquí que la coordinación misma distancia los mercados del mundo idealizado de la Competencia Perfecta, donde la organización industrial es virtualmente inexistente. Así, en palabras de Richardson: «no hay razón para esperar que las condiciones hipotéticas de mercado que 
definen la competencia perfecta podrían asegurar de hecho que la producción podría ser ejecutada del modo más eficiente, puesto que no existe razón para creer que el supuesto equilibrio podría ser alcanzado en algún momento. La conexión entre la estructura de mercado y el volumen de inversiones debe ser atribuida a los mecanismos particulares de ajuste, no en las supuestas posiciones de equilibrio con las cuales esa estructura se puede asociar. Aquí, igual que en otras partes, lo realmente importante se ha visto privado del análisis que requiere como resultado de la tiranía que el concepto de equilibrio ha ejercido sobre la teoría económica moderna.» ${ }^{22}$ La organización económica, los arreglos entre empresas, la coordinación y la cooperación entre empresarios son esenciales para que la información pueda fluir entre los agentes económicos. Pero al mismo tiempo, tales arreglos hacen realidad un distanciamiento del equilibrio óptimo. Esta crítica es igualmente aplicable a los modelos de competencia imperfecta que aprehenden la realidad desde una perspectiva de equilibrio; esto es, suponiendo que la información que es necesaria para alcanzar el punto óptimo ya está disponible a aquellos individuos que son parte de la ecuación incluida en el modelo. En consecuencia, la crítica es igualmente aplicable a la supuesta eficacia del modelo de competencia efectiva y al propio modelo de monopolio puro, como referencias a ser utilizadas para calibrar los mercados reales.

Con todo, el modelo de Competencia Perfecta «permaneció sin duda, para mucha gente, como un ideal o forma de organización modelo-en sentido estricto una categoría lógica, por oposición a un ideal normativo, aunque esta distinción no siempre fue hecha apropiadamente. No parecía haber sido reconocido que el hecho de que las 'imperfecciones', en algún grado o forma, sean claramente un obstáculo al ajuste, no debiera

22 Richardson (1960), p. 89. 
llevar a la conclusión de que sería mejor si tales imperfecciones no existieran. Sin embargo la conveniencia pedagógica de la Competencia Perfecta, y su adecuación como fundamento para una elaboración formal y matemática ulterior, dio al sistema un lugar central en la discusión teórica.» ${ }^{23}$ En suma, evaluar el funcionamiento de los mercados con un estándar de perfección ideal representado por la Competencia Perfecta es no sólo ingenuo (pues supone que tal información está disponible para el investigador); es también engañoso, ya que le estaría diciendo al regulador muy poco sobre la verdadera naturaleza del comportamiento que debe normar. Lo cierto es que no vivimos en un mundo de perfección (o imperfección), sino en un mundo donde los individuos deben asumir costes a veces onerosos, pero siempre necesarios a fin de poder alcanzar sus metas. Es imposible para los reguladores establecer apreciaciones normativas en determinados momentos instantáneos de la evolución de los mercados, ya que la realidad constantemente cambia porque el tiempo es irreversible. Permanentemente, nueva información altera las conclusiones normativas que los reguladores construyen en relación con un momento particular, sobre una supuesta condición óptima. En otras palabras, lo que aparece como óptimo en un momento determinado del tiempo, al minuto siguiente deja de serlo.

Como conclusión, puede afirmarse que la forma de pensar del Nirvana entraña un error intelectual fundamental: construye reglas morales sobre los mercados en base a una imagen de perfección que no puede existir en la realidad. No es éste sólo un ejercicio inútil, sino que además es un ejercicio peligroso. Es inútil, porque suponer que la realidad sería distinta si los reguladores fueran ángeles en vez de seres humanos, no contribuye a la tarea que una autoridad de la competencia debe llevar adelante, esto es, promover los intercambios de mercado,

23 Id., p. 39. 
y delimitar los derechos de propiedad. Pero aún más importante, es un ejercicio peligroso, ya que distrae y orienta la atención de los hacedores de políticas públicas hacia ejercicios erráticos de intervención pública basados en apreciaciones normativas dudosas, lo cual puede en algunos casos terminar siendo contraproducente cara al objeto de delimitar adecuadamente los derechos de propiedad. La comparación con la «perfección» solamente puede orientar a los responsables de las políticas públicas a juzgar equivocadamente el importante rol que juega la organización económica, los arreglos empresariales y la coordinación de las empresas, a fin de transmitir el conocimiento necesario a los actores del mercado para que puedan tomar decisiones de inversión. Tal perspectiva, hace aparecer tales arreglos como tentativas dolosas para manipular los mercados y alejarlos de la Competencia Perfecta, o cuando menos, Efectiva. Sin embargo, lejos del mundo ideal, la realidad es distinta.

El propio Schumpeter advirtió sobre el riesgo de que el regulador valore equivocadamente los mercados. Es sus palabras: «el problema que usualmente se toma en consideración es el de cómo administra el capitalismo las estructuras existentes, siendo así que el problema relevante es descubrir cómo las crea y como las destruye. Mientras no tenga conciencia de esto el investigador realiza una labor que carece de sentido; pero en cuanto lo reconozca, su visión de la práctica capitalista y sus consecuencias sociales se modificará considerablemente.» ${ }^{24}$ En otras palabras, el enfoque del Nirvana está equivocado desde un punto de vista epistemológico, ya que induce al analista a centrar su atención en los problemas de equilibrio de la asignación de recursos sociales, que son irrelevantes cara a los fines de entender de qué manera los mercados evolucionan endógenamente por medio de la competencia. Es claro que la

24 Schumpeter (1983), p. 122. 
visualización de los modelos convencionales de equilibrio entre los extremos de Monopolio Puro o Competencia Perfecta (por ejemplo, los modelos intermedios de Competencia Efectiva y Competencia Monopolística de E. Chamberlin y J. Robinson, respectivamente) no los hace menos estáticos y, por tanto, menos incapaces de aprehender la naturaleza evolutiva y creativa de la competencia.

Estos modelos supuestamente dinámicos, que dibujan las relaciones entre los agentes asociándolas con conductas estratégicas resultantes de asimetrías de información, no llegan a aprehender la naturaleza irreversible y evolutiva del mercado; por lo tanto, tales modelos fallan por su carácter estático y por su alejamiento del comportamiento de los agentes económicos en la realidad. Es muy revelador que la propia promotora de estos modelos, Joan Robinson, al reflexionar sobre la difícil coexistencia del análisis estático con la evolución temporal que caracteriza el trabajo de Marshall, comentara ya al final de su vida y en tono arrepentido, lo siguiente: «Cuando preparé Economics of Imperfect Competition sobre presupuestos estáticos, tome la ruta equivocada; el camino correcto hubiera sido abandonar el análisis estático (de equilibrio) y tratar de reconciliar el análisis con la Teoría del Desarrollo de Marshall.» ${ }^{25}$ Más tarde reafirmaría esta convicción: «tomé el camino equivocado en mi análisis de la competencia imperfecta, al concentrarme en lo 'imperfecto' e ignorar la 'competencia'. Así, en lugar de abandonar el análisis estático y tratar de reconciliar mi análisis con la Teoría del Desarrollo de Marshall, seguí a Pigou (quien desde hacía tiempo había desarrollado la esencia del análisis de Marshall dentro de un sistema lógico de teoría estática) y preparé Economics of Imperfect Competition sobre bases estáticas.» ${ }^{26}$ (Nuestras cursivas) Más claridad, imposible.

\footnotetext{
25 Robinson (1951).

26 Robinson (1951), p.viii.
} 


\section{V. \\ CONSECUENCIAS PRÁCTICAS DEL ENFOQUE DEL NIRVANA CONVENCIONAL EN LA APLICACIÓN DE POLÍTICAS PÚBLICAS}

La aplicación normativa del modelo de Competencia Perfecta (o Efectiva) en las políticas públicas ha resultado en lo siguiente: En primer lugar, en una perspectiva estrictamente jurídica, la comparación entre seres humanos «imperfectos» con el estándar de perfección óptima, conduce a la Autoridad de Competencia a desarrollar una aplicación inestable y ambigua de la política, erosionando el Estado de Derecho y la transparencia de las reglas de mercado. Así, cualquier nivel de cooperación entre los actores, será considerado sospechoso de encubrir una manipulación para desviar los mercados del equilibrio óptimo hacia las manos de los «monopolistas». Desde luego, siempre existe la posibilidad de demostrar que tal desviación acontece por razones de eficiencia económica, pero he aquí que tal análisis de eficiencia solamente puede ser calibrado por quienes aplican la política, y en consecuencia, cualquier otro sujeto (especialmente, las empresas investigadas) no tienen forma de anticipar si su acuerdo particular encajará dentro del estándar de eficiencia concebido en la mente del regulador.

La distinción entre comportamientos prohibidos «per se»y los sujetos a la «regla de la razón» no será muy útil para distinguir el comportamiento apropiado del inapropiado, pues ésta es una distinción estrictamente legal, no económica, cuyo propósito es evitar a la Autoridad de la Competencia los costes de examinar casos que, desde el punto de vista convencional parecen «obvios», tales como acuerdos horizontales de precios, que son considerados como prohibiciones «per se». Fácilmente se puede ver que ésta es otra forma de afirmar que el comportamiento prohibido "per se» siempre será prohibido 
porque es anticipadamente considerado - por efecto del análisis del Nirvana- como una conducta indeseable; no porque se sustente en un criterio económico que evalúe el impacto de dicha conducta en el mercado (lo que por su propia definición exigiría investigar las características del mercado, de los participantes, etc.). En otras palabras, es una tautología, que no nos ilustra - al igual que otras tautologías - sobre cuáles son las circunstancias fácticas que la Autoridad debe sopesar a fin de calificar un acuerdo como restrictivo sobre la base de consideraciones de eficiencia.

El estándar económico de eficiencia introduce de contrabando un análisis moral que incorpora las preferencias del regulador sobre la manera en que deben asignarse los recursos en la sociedad; por ser moral, tal parecer no puede estar sujeto a ninguna regla jurídica estable que incorpore las preferencias de los actores sociales, en uso de sus derechos de propiedad. Calibrar anticipadamente el criterio de la Autoridad puede ser poco menos que imposible, pues lo que hoy día encuentra como «eficiente» bien puede considerarlo como «ineficiente» mañana. En definitiva, sólo el funcionario de turno puede conocer en su mente cuál es el nivel particular de exigencia que habrá de comportar un escenario «óptimo» en un caso concreto.

Esta dificultad que tiene el investigador (o la Autoridad) para basar su criterio en una información factual precisa y objetiva es la que le induce a buscar información en factores aleatorios que no tienen que ver con los efectos mismos del comportamiento empresarial examinado, sino con la posición particular que, por azar, detente la empresa en el mercado relevante. De esta manera, las empresas con elevada cuota de mercado tenderán a ser vistas en esta película como los sospechosos de siempre.

En este sentido, el Premio Nobel de la universidad de Chicago, George Stigler, señaló que «las definiciones no arrojan 
ningún conocimiento sobre el mundo real, pero influencian las impresiones que pueden tenerse sobre el mundo. Si sólo los mercados con un gran número de comerciantes son 'perfectamente competitivos', y si los mercados con pocos comerciantes son llamados 'oligopolísticos' (literalmente, "pocos vendedores»), eso sugiere que estos últimos no son competitivos, ni tampoco perfectamente competitivos. La sospecha de los casos de mercados con pocos competidores fue gradualmente reforzada en los casos antimonopolios. ${ }^{27}$

No sorprende, por tanto, que la percepción sobre la ilegalidad haya cambiado tan dramáticamente en la jurisprudencia de los Estados Unidos y de la Unión Europea en la aplicación práctica de las políticas de competencia a lo largo de la Historia. Las conductas que anteriormente eran consideradas como ilegales «per se», hoy día son consideradas prácticas sujetas a la «regla de la razón»; las conductas que antes había sido perseguidas, hoy día son toleradas. Considérese la evolución de la aplicación de la política de precios mínimos de reventa, en la monopolización de los mercados, o la política de fusiones y adquisiciones. En todos estos ámbitos el cambio de la jurisprudencia ha sido notable.

Desde luego, no es sano cargar excesivamente las tintas. No todo ha sido anarquía en la aplicación de las normas de competencia. El argumento esbozado aquí simplemente pretende sugerir que si ha habido estabilidad en la aplicación de las normas de competencia a lo largo del tiempo, ello se debe a otros factores institucionales que han servido para limitar el campo de acción de las Autoridades de la Competencia en la aplicación de su política. La existencia de una cierta estabilidad en la teoría económica dominante acerca de los mercados, aunada a otros valores en el ordenamiento jurídico, tales como el respeto a los derechos de propiedad y el derecho a la defensa,

27 Stigler (1988), p. 94. 
han propiciado una cierta predictibilidad en la aplicación de las políticas de competencia. La política de competencia ha sido relativamente estable en los países industrializados, pese a sus postulados descoordinadores, gracias a la solidez de sus principios de Derecho que conforman sus instituciones. Igualmente, hay que dar cierto crédito al hecho de que hay cierta estabilidad en la teoría económica producto de la convención profesional, a lo largo de periodos más o menos extendidos, que ha contribuido a dar un cierto aire de respetabilidad al sistema.

Con todo, es preciso destacar que esta erosión del Estado de Derecho es particularmente perniciosa en el caso de los países en desarrollo. En estos países la construcción institucional de las reglas de mercado es una tarea fundamental donde el enfoque convencional, lejos de contribuir a tal propósito, se convierte en un poderoso instrumento de erosión de las expectativas de los agentes económicos en el mercado, reforzando una tendencia discrecional a favor de la Autoridad, que es absolutamente indeseable de la promoción de los mercados de tales países. La estabilidad institucional, a diferencia de lo que sucede en los países industrializados, no puede darse por supuesta en los países en desarrollo. La transición misma de estas economías fuertemente reguladas hacia el Mercado introduce por sí misma una gran incertidumbre entre los agentes económicos, que está ausente de las jurisdicciones de los países desarrollados, para quienes estos problemas están prácticamente resueltos. Esta es una consideración que frecuentemente se pasa por alto, especialmente por aquellos que promueven la conveniencia de adoptar políticas de competencia en países en desarrollo, sin examinar el marco institucional en el cual dicha política debe insertarse. Esta incertidumbre en la aplicación de las políticas refuerza la incertidumbre que, de entrada, ya poseen las empresas sobre el ambiente en el cual deben invertir. 
Por otro lado, al concentrarse en la posición estática alcanzada por los mercados en un momento dado de su evolución, es inevitable que el analista enfoque su interés en las consecuencias sobre el bienestar social, consideración ésta que tiene que ver con la asignación de los recursos hecha «desde lo Alto» por el regulador, y no sobre la base del funcionamiento de los mercados que se materializa en las decisiones de los individuos que participan en él. Por lo tanto, se trata de una preocupación que virtualmente excluye del análisis toda consideración acerca del rol institucional que desempeñan los mercados. Como se indicó anteriormente, eso no es casualidad, ya que, bajo dicho análisis, el marco institucional se da por sentado ex definitione. No obstante, es precisamente el problema de la construcción institucional el que tiene que más tiene que ver con la necesaria creación de las condiciones para el desarrollo en los países en transición.

VI.

\section{LA ESTRUCTURA EVOLUTIVA DE LAS EXPECTATIVAS EN LOS MERCADOS}

Para regular los mercados adecuadamente, es preciso comprender su funcionamiento. Por ello, hay que partir de una perspectiva que asuma, no sólo la condición naturalmente desinformada de los agentes del mercado, sino también del investigador o analista. ${ }^{28}$ De modo que el problema económico no está ligado al uso

28 Como señalara agudamente Loasby (1991), p. 2, esta premisa cognitiva no es exclusiva de la ciencia económica. En sus palabras: «Quisiera atraer la atención de los lectores a la semejanza fundamental de los problemas que confrontan los economistas y los agentes económicos que pretenden estudiar. Ambos grupos tratan de dar sentido al mundo en el que se encuentran, y de comportarse inteligentemente en él (paralelismos similares entre los practicantes de una disciplina y sus sujetos de estudio se encuentran en otras ciencias humanas). De modo que 
de los recursos sociales, que pueda ser resuelto por un tercero capaz de obtener la información necesaria para guiar los agentes económicos hacia el Bienestar Social. Más bien está relacionado con la coordinación de las actividades de los agentes económicos para superar su incertidumbre en el mercado.

La actividad de los individuos está marcada por una «niebla de ignorancia», lo que significa que deben conformarse con hacer especulaciones acerca del futuro. En esta difícil tarea de anticipar el futuro, los individuos pueden guiarse por sus intuiciones, que dependen en parte de su experiencia pasada en circunstancias similares; de su experiencia, pueden aprender a relacionar ciertas tendencias o identificar ciertos resultados futuros posibles. Sin embargo, en la preparación de sus planes, nunca podrán tener completa certeza; así, están confrontados inexorablemente a un problema insoluble de incertidumbre radical.

Lo importante es que en este contexto especulativo, los empresarios están forzados a buscar información suficiente sobre eventos futuros que les motive a invertir hoy. Esto es lo fundamental, pues tal inversión es la que determina el nivel futuro de producción. En un sistema donde las decisiones están descentralizadas (en un sistema de mercado), cada individuo ignora las acciones futuras de los demás; así, sus propias acciones no son más que conjeturas (hipótesis) sobre un futuro incierto. El significado de esas conjeturas se encuentra mucho más allá de lo que pudiera ser considerado como una simple especulación acerca de la posible ocurrencia de un evento. Los cálculos actuariales, por ejemplo, establecen la regularidad por la cual ciertos eventos pueden llegar a suceder, lo que permite a los empresarios reducir («internalizar») el posible coste y riesgo.

el comportamiento de los economistas puede ayudarnos a comprender el comportamiento de los agentes económicos y viceversa.» 
Pero he aquí que no nos estamos refiriendo a esa clase de eventos futuros, que constituyen el objeto de los seguros contra los riesgos futuros. ${ }^{29}$ A diferencia del riesgo, la incertidumbre radical de los mercados se refiere a un evento absolutamente impredecible, porque tiene que ver con las conjeturas de los seres humanos; no con la ocurrencia de eventos de la naturaleza.

Fue Keynes quien propuso el mejor ejemplo de incertidumbre radical, en su conocido caso del "Concurso de Belleza». La esencia especulativa de las decisiones que adoptan los agentes en el mercado es semejante a la de un concurso de belleza donde la votación ha de resolverse, no teniendo en cuenta la candidata que los jueces juzgan más bonita o talentosa, sino considerando la candidata que cada juez cree que será la más votada por los demás jueces. La ganadora será aquella escogida de acuerdo con las expectativas recíprocas de los jueces. En efecto, como quiera que la decisión de cada juez depende de lo que él cree que otros miembros del jurado decidirán, pero sin tener absoluta seguridad de ello (obviamente, si no media comunicación entre ellos), por basarse en las expectativas subjetivas de éstos, y no en hechos objetivos, su decisión estará basada en la incertidumbre sobre quién ganará. ${ }^{30} \mathrm{La}$ votación no tendrá fin, pues será un proceso de conjeturas sobre lo que decidirá el otro, a su vez determinada por la conjetura sobre qué decidirá el primero. La única forma de terminar con esa inmovilidad paralizante es apelando a la decisión de una autoridad superior, o resolver el problema mediante convención entre los jueces (o participantes en el mercado), permitiéndoles, por ejemplo, que se puedan comunicar entre sí.

29 Ésta es la distinción entre «riesgo»e «incertidumbre» a la que se refiere Frank Knight (1921), en su obra Risk, Uncertainty and Profit.

30 Keynes (1936 [1964]), p. 156. 
Como han indicado O'Driscoll y Rizzo: «En principio, no existe límite al nivel de formulación de conjeturas recíprocas. No existe razón lógica suficiente para detenerse en algún punto en particular; detenerse es, en buena medida, arbitrario o resultado de convención.» ${ }^{31}$ En el caso de transacciones de mercado, estas convenciones algunas veces adoptan la forma de cooperación explícita a través de variados acuerdos y arreglos, o mediante entendimiento tácito. También influyen la reputación, la práctica comercial previa y otras realidades semejantes.

Si el problema de las empresas es la coordinación, y ella está limitada por la incertidumbre radical, ¿cuál es el objetivo de la política pública orientada a promover la competencia?

\section{VII. \\ EL NUEVO ENFOQUE DE LOS MERCADOS Y LA COMPETENCIA}

El interés por calibrar mejor el rol que juegan los mecanismos de transmisión de información que las empresas utilizan para aprovechar oportunidades en los mercados, ha moderado el rigor que tenía la política de competencia ante tales mecanismos, ampliando el rango de conductas consideradas ilegales solamente cuando se demuestra un efecto anticompetitivo en el mercado, y limitando el espectro de conductas consideradas per se socialmente negativas.

El nuevo enfoque se asienta en la idea de que la competencia surge de un proceso de "creación destructiva», donde la capacidad para participar en el mercado depende de la creatividad, innovación, y posicionamiento estratégico de las capacidades empresariales para procesar información de los

31 O’Driscoll y Rizzo (1985), p. 73. 
consumidores y productores que constantemente fluye en el mercado y que ha de ser correctamente interpretada por las empresas en el momento en que tomen sus decisiones empresariales. El contexto determinante de la actividad empresarial está caracterizado por la incertidumbre y la especulación. Las firmas están constantemente forzadas a medir el nivel de los recursos que deben dedicar hoy para alcanzar un nivel esperado de demanda agregada en el futuro. El problema surge, sin embargo, porque cualquier determinación acerca de los futuros niveles de demanda agregada será, por definición, especulativa. No obstante, las empresas deben tomar una decisión al respecto, bien sea para invertir o para decidir no hacerlo. ${ }^{32}$ Éste es un proceso de prueba y error que las fuerza constantemente a experimentar con diferentes alternativas de inversión.

Obviamente, estas decisiones jamás son idénticas entre sí, ya que las circunstancias que las determinan cambian. La condición de empresario demanda por ello que se preste atención a las nuevas oportunidades, que los empresarios estén «alertas».33 Serán tales nuevas circunstancias las que les «dirán» que ciertas tecnologías o técnicas de producción prevalecerán, y que otras serán reemplazadas, o que los consumidores cambiarán sus preferencias, o que las mantendrán. En suma, las nuevas circunstancias colocan al empresario ante el dilema de aumentar o disminuir sus niveles de inversión en el presente, para corresponder a los niveles de demanda agregada futura.

¿De qué manera sucede esto? Tal como señala Richardson, al tomar sus decisiones, los empresarios deben confrontar su incertidumbre, y los arreglos institucionales que negocian con otros empresarios, según el tipo de inversiones que realizan; las competitivas y las complementarias.

\footnotetext{
${ }^{32}$ Richardson (1960), pp. 29-46.

33 Kirzner (1973).
} 
En el primer caso, el incremento de la inversión competitiva de un empresario limita negativamente las posibilidades de que los demás coloquen su propia producción en el mercado. Cada empresario compite por producir más, para copar la capacidad de demanda agregada del mercado, pero ello desplaza las posibilidades de los demás competidores.Aunque todos aspiran a un pedazo de dicha demanda agregada futura, dado que la asignación final es incierta, el resultado será que hoy tendrán menos incentivos para invertir.

Richardson compara la situación de las inversiones competitivas con la de varios participantes en una lotería en la que el premio se obtendrá siempre que el nivel total de lo jugado por los apostadores no exceda una cierta suma, pues en caso de exceso, cada jugador deberá soportar una pérdida proporcional. De igual modo, en el mercado, la suma colectiva de las inversiones competitivas de hoy, hecha individualmente y sin contacto alguno con los demás participantes por cada uno de los competidores, no puede generar en el futuro una producción conjunta superior a la cantidad total de demanda agregada que se espera en el mercado. En caso de exceso, cada uno deberá soportar pérdidas proporcionales a lo invertido en exceso. La pregunta es: ¿cómo pueden asegurarse los empresarios de que invertirán hoy la cantidad máxima óptima? En un escenario de mercado donde las decisiones se toman descentralizadamente y sin contacto entre los participantes, cada agente económico debe depender de sus propias expectativas, que, como hemos visto en el Concurso de Belleza, conducen a una regresión infinita de especulación acerca de lo que harán los demás en el mercado, esto es, a la incertidumbre total.

En el segundo caso, el incremento de una inversión complementaria debe ser suficiente para inducir a los demás a realizar inversiones complementarias a la primera. El problema entonces es: ¿cómo pueden asegurarse los empresarios de que 
otros invertirán hoy la cantidad mínima? La respuesta es, en uno y otro caso, la cooperación empresarial y el seguimiento de las acciones de los demás empresarios en el mercado. Los empresarios cooperan para reducir el desperdicio que resulta de equivocaciones en que pudieran incurrir, debido a conjeturas mal hechas o a informaciones incompletas. Para lograrlo, entran en un proceso de ensayo y error que les permite «descubrir» cuales de sus expectativas iniciales vale la pena preservar, en tanto que corresponden con los hechos que con el tiempo se verifican (y el futuro se vuelve presente).

Los empresarios construyen expectativas mutuas a través de ciertos arreglos dirigidos a dotarlos de ciertas seguridades sobre sus futuras situaciones. Por ejemplo, pueden apoyarse en su experiencia comercial pasada; también, pueden basar su juicio sobre la reputación de las firmas con las que negocian o compiten (lo que, a su vez, refleja la experiencia comercial de esas empresas con terceros); finalmente, pueden recurrir a arreglos convencionales formalizados en contratos, como vía para asegurar el cumplimiento de las expectativas creadas; si no es posible la vía contractual, las partes buscarán alinear su conducta con la de otros empresarios, sobre la base de lo que esperan de ellos, a través de arreglos informales como «acuerdos de caballeros», y así sucesivamente. Estos arreglos institucionales, así como muchos otros, aunque son menos confiables que suscribir un contrato de seguro, proveen sin embargo a los empresarios que participan en ellos de la suficiente certeza para realizar inversiones hoy que afectarán sus niveles de producción futura.

Obviamente, todas esas técnicas limitan a los empresarios las posibilidades de adoptar cursos «independientes» de acción; sin embargo, ha de notarse que la limitación introducida no es un resultado libremente escogido de monopolizar mercados, sino más bien la necesidad inevitable e imperiosa de coordinar actividades dirigidas a prevenir pérdidas futuras debido 
a errores en la predicción. Dicho de otra manera, los empresarios no tienen libertad de acción real al decidir optar por alinear su conducta con la de los demás, por cuanto esa es la única manera que tienen de conocer si deben invertir o no.

Además, tal cooperación no excluye en sí misma la rivalidad, pues de hecho el futuro es incierto, y tales acuerdos están constantemente sujetos a tensión como resultado de los cambios imprevistos que las nuevas circunstancias introducen en las relaciones de mercado. Son esos cambios los que estimulan a los empresarios a introducirse en sectores antes no explorados, a innovar, a ser los primeros en desarrollar ciertos productos o tecnologías, a actuar independientemente de la convención establecida por el grupo, en la expectativa de obtener un beneficio más elevado que en cooperación con otros, etc. Los cambios inesperados crean nuevas oportunidades, y abren vacíos de conocimiento que deben ser llenados, y de hecho son llenados, por empresarios agresivos y alertas en búsqueda de ganancias. Es esto lo que induce a otros seguirlos, bajo la estructura de relaciones de coordinación, a fin de capitalizar las oportunidades que las empresas van descubriendo en el mercado.

Bajo el nuevo enfoque, por lo tanto, no todas las estrategias realizadas por una o más empresas tienen como objetivo poner en desventaja a sus competidores; por el contrario en muchas ocasiones dicha situación es el resultado inevitable de las acciones llevadas a cabo por una empresa con el fin de reducir sus costes de producción y distribución, mejorar la calidad de sus productos $u$ otros fines que se encuentran dentro de la dinámica del proceso competitivo. En ocasiones la tarea más difícil a la que deben hacer frente las agencias de competencia, es fijar el límite entre aquello que va en pro de la eficiencia en el mercado y aquellas situaciones en las que el objetivo es dañar al competidor o a la competencia. Quizás el factor que dificulta en mayor grado tal proceso, radique en el hecho de 
que las consecuencias de una determinada estrategia sólo son notorias a largo plazo, razón por la cual las agencias de competencia en ocasiones deben prever lo que puede ocurrir en el futuro, a partir del flujo de información que manejen en el presente.

De acuerdo con esta perspectiva, la permanencia de carteles o la consolidación de posiciones dominantes en el mercado son fenómenos transitorios, que son desmontados gracias a la innovación que se produce en el mercado. De acuerdo con esta idea, no interesa tanto reprimir posiciones dominantes como permitir que se produzca la innovación. En este enfoque moderno, la política de competencia se visualiza esencialmente como un catalizador de las capacidades empresariales dirigidas a complementar y promover la competitividad de las empresas que participan en el comercio internacional, más que como un instrumento para asignar los recursos económicos entre los distintos actores económicos, conforme a una fórmula estática de eficiencia social «óptima».

VIII.

LA RECEPCIÓN DE LAS IDEAS SOBRE LA COMPETENCIA EN LATINOAMÉRICA

De esta manera, siendo inestable desde el ángulo epistemológico el terreno en el cual se asienta la percepción convencional de los mercados, es lógico suponer que el edificio constituido por los conceptos creados para interpretar el comportamiento de las empresas en los mismos, esté sujeto al mismo fallo.

Así, la explicación «estructural» de la competencia, que visualiza la competencia de los mercados reales a partir del modelo «desconcentrado» de la competencia perfecta o el «concentrado» de monopolio y oligopolio, está siendo objeto de reconsideración en la doctrina económica. 
Naturalmente ello está afectando la práctica de la política de la competencia, si bien a ritmos distintos, según el grado de apercibimiento de la autoridad, sobre la relevancia de la crítica hecha al pensamiento convencional, y la aceptación entre los reguladores de las nuevas corrientes de pensamiento en torno a los mercados y al fenómeno económico de la competencia, vistos como procesos de adaptación al cambio del entorno en el cual actúan las empresas en vez de estructuras estáticas cercanas o lejanas (según sea el caso) a modelos de competencia perfecta o monopolio.

Pero el proceso de adopción de las nuevas ideas ha sido lento, y quizás más intuitivo que consciente, ante la necesidad de dar solución a los problemas prácticos de promover la capacidad de las empresas para competir internacionalmente, lo que ha hecho necesario considerar problemas asociados con la competitividad del «marco» en el cual las empresas compiten domésticamente.

Desde luego, ha sido considerable el peso institucional ejercido por las políticas públicas intervencionistas tradicionalmente aplicadas en la Región. Así, al apreciar los desarrollos jurisprudenciales en diez años de aplicación de políticas de competencia, se observa que el desarrollo de la regulación antimonopolio en América Latina ha estado asociado tradicionalmente con una visión estructural y estática de los mercados, tendente a promover la intervención estatal para modular los efectos «negativos» de los arreglos corporativos empresariales, que son percibidos de este modo como expresiones de comportamiento monopolístico, necesitado de control estatal.

Si se examina más de cerca esta percepción del problema de los mercados, se verá en ella una tendencia de continuidad con la visualización dirigista de los mercados que fue tradicionalmente aplicada en Latinoamérica antes de la apertura económica de la década de los ochenta, si bien bajo la coartada 
de promover «la asignación eficiente de los recursos» en lugar de las razones de «desarrollo» esgrimidas hasta entonces, pero compartiendo resultados similares, a saber, la alteración del curso natural de los mercados, y por supuesto, de la dinámica de la competencia.

Éste es un dato crucial para comprender el sentido de la agenda de las políticas de competencia aplicadas en la región, pues por tal motivo, puede afirmarse que el paradigma utilizado para acercarse a la comprensión de las instituciones sociales (entre ellas, el mercado), entre quienes apoyan políticas antimonopolio ha sido en esencia el mismo antes y después de la apertura económica de la Región. Así, de las políticas de fomento industrial que ensalzaban la redistribución equitativa de los recursos sociales con miras a fortalecer la capacidad de desarrollo de sectores o grupos menos favorecidos, sectores estratégicos y «campeones nacionales», se ha pasado a políticas de fomento de la competencia cuya premisa orientadora, esto es, la eficiencia, impone un criterio de asignación «óptima», en función del cual, paradójicamente, debe sacrificarse la propia asignación espontánea ocurrida en el mercado, si el regulador estima que su visión de la eficiencia del sistema es distinta de la asignación que cabría esperar de la dinámica del mercado.

De esta manera, si bien es indudable que la idea de que los mercados suelen asignar los recursos sociales de manera más eficiente que la planificación impuesta desde el Estado, no cabría esperar que la formulación de políticas públicas fuese muy distinta después de iniciada la reforma, pese a que poco a poco ha ido calando entre la intelectualidad.

La «forma de comprender» los mercados y la competencia condujo a una particular concepción acerca de los casos de conductas y prácticas que habrían de tolerarse y los que habrían de prohibirse. Pese a no ser propia de la cultura jurídica latinoamericana, la distinción entre conductas prohibidas per se y 
conductas sujetas a la regla de la razón fue casi unánimemente adoptada por las diversas autoridades de competencia de la Región. De igual modo, el control de fusiones y adquisiciones, que nunca antes había sido aplicado por autoridad alguna, ha pasado a ser uno de los instrumentos preferidos por estas autoridades en la supervisión de los mercados, siendo que el mismo es la síntesis de la negación misma de la dinámica espontánea de los mercados.

Así, este paradigma explicativo de la visión tenida en las relaciones de mercado, y de la intervención correctiva de la autoridad sobre sus supuestos «fallos», vino a ser el dominante a partir del momento en que se inició la reforma económica que concluyó en la apertura económica de América Latina, en la década de los ochenta.

Como se dijo antes, esta visión de los mercados y de la competencia ha cambiado en la teoría económica, ante la incapacidad de la postura convencional para atribuir un rol a la coordinación empresarial que se produce en los mercados, distinto del fácil recurso de calificarlas como una forma de «fallo» que aleja a los mercados «reales» del modelo de Competencia Perfecta.

IX.

\section{EL DIVORCIO POSITIVISTA ENTRE EL DERECHO Y LA ECONOMÍA}

En el proceso antes descrito se observa un fenómeno de mayor alcance, que merece atención, esto es, la disposición de los juristas a tomar partido por criterios normativos surgidos de la ciencia económica, como es el caso de la eficiencia. Éste es un fenómeno que revela tanto el «imperialismo» de la ciencia económica sobre otras ciencias sociales, ${ }^{34}$ como la inca-

${ }^{34}$ Como ha descrito Lazear (1999), en los siguientes términos: «El enfoque en la eficiencia ha hecho que los economistas centren su atención sobre problemas que 
pacidad de la ciencia jurídica para ofrecer una alternativa atractiva.

Desde luego, este fenómeno es producto de la renuncia positivista a examinar conceptos normativos considerados por ésta como «metafísicos», «moralistas» $\mathrm{y}$ "anticientíficos», como es el caso de la «justicia», los «derechos subjetivos» o la «propiedad individual», todos los cuales encontraban su explicación en la moral y no en la «ciencia jurídica». Con el avance de la ingeniería social estos conceptos transmutaron en otros, por medio de adjetivos que no hicieron más que desnaturalizar su contenido: justicia «social», derechos «colectivos», etcétera.

Por su parte, la ciencia económica neoclásica, al poseer modelos cuantitativos presuntamente capaces de "predecir» el comportamiento de los agentes económicos, se encontraba aparentemente mejor posicionada que las demás ciencias sociales para proveer las valoraciones normativas «genuinamente científicas» que requería el análisis jurídico para poder hacerse efectivo en la práctica. Con la visión matemática y formal de la economía neoclásica positivista, los individuos pueden ser representados como perfectamente egoístas y obsesivamente orientados a maximizar su utilidad (homo economicus). Bajo un cálculo de agregación de preferencias individuales, sería posible para el analista neoclásico deducir una fórmula de bienestar social, a partir de un cálculo de costebeneficio típicamente utilitarista. ${ }^{35}$ La moral utilitarista terminó

otras ciencias sociales prefieren dejar de lado. Estos ingredientes han permitido a los economistas invadir un territorio intelectual que anteriormente había sido considerado ajeno al campo de dicha disciplina.»

35 Sobre esta supuesta «neutralidad» analítica pregonada por el positivismo en las ciencias sociales, Capra (1992, p. 216) correctamente señala: «Cualquier análisis "desprovisto de valores" de un fenómeno social se basa en la suposición tácita de que existe un sistema de valores implícito en la selección y la interpretación de datos. Evitar el tema de los valores, pues, no significa que los especialistas en ciencias sociales sean más científicos, sino que, por el contrario, están siendo menos 
por consolidarse en el ámbito de la ciencia económica. Por su parte, el Derecho dejó de ser «normativo» para convertirse en «funcional» o «instrumental», 36 donde el objetivo del instrumento era, desde luego, aportado por una ciencia social distinta al Derecho, esto es, alcanzar la eficiencia, dentro de la estrechez que la escuela neoclásica de la economía había atribuido a dicho concepto.

Eventualmente, la desaparición del sustrato «metafísico» que servía de sustento jurídico al orden del mercado, y su reemplazo por una ética utilitarista supuso desarticular las defensas del orden jurídico frente al avance arrollador del intervencionismo estatal inspirado en los conceptos de la economía neoclásica («fallos de mercado», «externalidades», etc.). Los derechos individuales comenzaron a verse como impedimentos o molestias a la materialización de la ingeniería social, guiada por el bienestar social definido por el cálculo utilitarista.

Es claro que la consecución de un verdadero orden de mercado no interferido por la ingeniería social, está inspirado en la equidad y distribución de los recursos, o en la eficiencia, requerirá un profundo examen de los fines del Derecho. Esto es algo que está comenzando a suceder. Notando la tranquila aceptación de los juristas ante la ingeniería social positivista, Hayek observaba ya a mediados de la década de los ochenta lo siguiente: «dudoso resulta que tal avalancha de falsa ciencia económica haya tenido a lo largo del último siglo vía de difusión más propicia que las lecciones impartidas en las aulas universitarias a las nuevas generaciones de juristas, ante quienes se ha pregonado que 'era necesario' que se hiciera esto o aquello, o que determinadas circunstancias 'hacían inevitable'

científicos, al no exponer explícitamente las suposiciones en las que se apoyan sus teorías.»

36 Loughlin (1992). 
la adopción de tales o cuales medidas. Para el hombre de leyes resulta casi hábito normal pensar que si el legislador ha decidido algo, huelga la necesidad de toda ulterior comprobación acerca del acierto de su decisión. Quiere esto decir que su labor será beneficiosa o perniciosa según la bondad o la falsedad de los precedentes que la orienten, por lo que tanta probabilidad existe de que se perpetúen pretéritos errores como que se renueven pasados aciertos. En la medida en que acepte como preceptivas las tendencias imperantes, el legislador puede convertirse tanto en simple instrumento de la introducción de intervenciones cuyo contenido no comprende como en creador consciente de un nuevo orden. Todo ello subraya la necesidad de buscar fuera de la ciencia jurídica aquellos criterios que adecuadamente permitan enjuiciar las materias que nos ocupan.»37

Que esta mansedumbre ante la ingeniería social es producto de la desarticulación de las defensas metafísicas del Derecho, es algo que está comenzando a reconocerse. Así, por ejemplo, García de Enterría se ha referido a los excesos del positivismo jurídico en la creación de lo que es calificado por él como «absolutismo legislativo regulador y opresor» ${ }^{38}$ Será

37 Hayek (1973 [1985]), 134.

38 En sus palabras: «Hoy, dos siglos después (del triunfo del concepto positivista en el Derecho Revolucionario Francés), nuestra fe en la Ley está, ciertamente, bastante quebrantada. De Rousseau, buscando su suelo liberador, hemos venido a recaer, inesperadamente, en Hobbes, en la expresiva fórmula de Dahrendorf, en un absolutismo legislativo regulador y opresor, a su vez generador de incertidumbre y de anomia moral, y no propiamente en el reino de la libertad.» (Op. cit, 124) Con todo, García de Enterría termina por aceptar el estado de cosas, al reconocer que: «no parece que tengamos ya otra alternativa que la de seguir rigiéndonos por Leyes. Todo el problema es ahora mejorar su calidad (tanto en sí misma como respecto a su servicio a unos derechos fundamentales, cuya súper legalidad ya no se niega), renunciar a creer que los preceptos escritos pueden encerrar en sus mallas la totalidad de la casuística de la vida social, la cual, además, está afectada de un proceso de cambio y evolución constantes, y, correlativamente, en la técnica aplicada de la Ley aceptar que pueden entrar en juego otras fuentes del Derecho, especialmente los principios generales del Derecho.»(Op. cit.) 
preciso redefinir el paradigma ${ }^{39}$ bajo el cual ha sido concebida la ciencia jurídica contemporánea, reivindicando la obvia asociación entre la ciencia económica y la jurídica, como dos caras complementarias de la realidad social, en igualdad de condiciones y méritos, sin que sea posible aceptar la influencia imperialista de una sobre la otra.

\section{$X$. CONCLUSIÓN}

En la visión convencional de la teoría de la organización industrial se afirma que los fallos de mercado son responsables de la asignación sub-óptima de los recursos sociales. El comportamiento monopolístico hace que dichos mercados incurran en fallos, en tanto dicho comportamiento permite la creación de condiciones en las cuales las asimetrías de información se explotan a favor de los presuntos monopolistas. Como consecuencia, la eficiencia del mercado será inferior a la que habría en condiciones óptimas establecidas por el modelo de Competencia Perfecta, donde los productos son indiferenciados, la información fluye libremente y las firmas son tomadoras de precios, en vez de «manipuladoras» de precios.

En consecuencia, la esencia de esta perspectiva está anclada en la comparación entre el modelo ideal de perfección construido en la mente, por una parte y la imperfección de la realidad que percibimos a través de nuestros sentidos, por otra parte. Este es un ejercicio intelectual dudoso, pues compara la realidad con un estándar de perfección ideal que, bajo sus propios presupuestos, deja fuera los costes que los individuos deben soportar a fin de alcanzarlo. Es decir, realiza una

39 Utilizamos la expresión "paradigma», en el sentido acuñado por Kuhn (1970). 
comparación entre dos categorías distintas e incompatibles de la mente, por una parte, una categoría ideal de un estado óptimo, y por la otra, una evaluación sensorial de la realidad.

Como señala McNulty, «que la competencia perfecta es un ideal, incapaz de ser materializado en la realidad, es ya un tema familiar en la literatura económica. Que por varias razones sería menos que deseable, incluso aunque fuese realizable ha sido también reconocido. Pero que la competencia perfecta es un estado de cosas completamente incompatible con la idea de cualquier (y todo) tipo de competencia ha sido insuficientemente enfatizado. ${ }^{40}$

La aplicación de políticas de competencia ha de reconocer los problemas derivados de esta incompatibilidad, a fin de orientar sus objetivos de política hacia la construcción institucional de los mercados, no a su destrucción, en nombre de la competencia.

\section{BIBLIOGRAFÍA}

Baumol, W.; PAnZar, R. y Willig, R. (1982), Contestable Markets and the Theory of Market Structure, Harcourt Brace Javanovich, Inc., Nueva York.

Bork, R. (1978), The Antitrust Paradox: A Policy at War with Itself, Basic Books Publisher, Nueva York.

Burton, J. (1994), «Competition over Competition Analysis: A guide to some Contemporary Economics Disputes», in Frontiers of Competition Law (Birmingham: Institute of European Law).

Caves, R. y Porter, M. (1977), «From Entry Barriers to Mobility Barriers: Conjectural Decisions and Contrived Deterrence

40 McNulty (1975, pp. 65-66). 
to New Competition», Quarterly Journal of Economics, mayo, 241- 262.

Clark, J.M. (1940), «Toward a Concept of Workable Competition», The American Economic Review, 30, junio.

Crampton, P. (2004), "Competition and Efficiency as Organizing Principles for All Economic and Regulatory Policymaking», Working paper n. ${ }^{\circ}$ 2, Interamerican Development Bank, enero.

Demsetz, H. (1969), «Information and Efficiency: Another Viewpoint», Journal of Law and Economics, 12, pp. 1-22.

- (1982), Economic, Legal and Political Dimensions of Competition, Professor Dr. F. de Vries, Lectures in Economies, v. 4, North-Holland, Nueva York, pp. 386-393.

Dorfman, R.P.; SAMuelson, P. y Solow, R.M. (1958), Linear Programming and Economic Analysis, McGraw Hill, Nueva York.

Epstein, R. (1995), Simple Rules for a Complex World, Harvard University Press, Cambridge.

Friedman, M. (1953), "On the Methodology of Positive Economics», Essays in Positive Economics, M. Friedman (ed.), University of Chicago Press, Chicago.

HAYEK, F. A. (1948), Individualism and Economic Order, The University of Chicago Press, Routledge, Londres.

- $\quad$ (1973 [1985]), Derecho, Legislación y Libertad (Vol. 1), Unión Editorial, Madrid.

- (1973), Law, Legislation and Liberty, Vol. 1, University of Chicago Press, Chicago.

- (1976), Law, Legislation and Liberty: The Political Order of a Free People, Vol. 3, University of Chicago Press, Chicago.

KeYnes, J.M. (1936 [1964]), The General Theory of Employment, Interest and Money, Nueva York: Harcourt, Brace \& World.

KIRZNER, I. (1973), Competition and Entrepreneurship, University of Chicago Press, Chicago. 
KLEIN, B. (1990), «The Use of Economics in Anti-trust Litigation: Realistic Models of the Competitive Process», in The Law and Economics of Competition Policy, F. Mathewson et al. [eds.] (Vancouver: The Fraser Institute.)

KNight, F. (1921), Risk, Uncertainty and Profit, Boston, MA: Hart, Schaffner \& Marx; Houghton Mifflin Company.

Kunn, T. (1970), The Structure of Scientific Revolutions, The University of Chicago Press, Chicago.

LAZEAR, E. (1999), «Economic Imperialism», Working Paper 7300, National Economic Bureau of Economic Research, Cambridge.

LOASBy, B. (1991), Equilibrium and Evolution: An exploration of connecting principles in economics, Manchester University Press, Manchester.

Loughlin, M. (1992), Public Law and Political Theory, Clarendon Press, Oxford.

McNulty, P. (1975), «Economic Theory and the Meaning of Competition», en The Competitive Economy, Y. Brozen (ed.), General Learning Press, Morristown, Nueva York.

O'Driscoll, G. y Rizzo, M. (1985), The Economics of Time and Ignorance, Basil Blackwell, Oxford.

Popper, K. (1990), «Hacia una Teoría Evolutiva del Conocimiento», en Un Mundo de Propensiones, Editorial Tecnos, Madrid.

Richardson, G.B. (1960), Information and Investment, Oxford University Press, Londres.

Robinson, J. (1951), Collected Economic Papers, Vol. 1, Basil Blackwell, Oxford.

Schumpeter, J. (1983), Capitalismo, Socialismo y Democracia, Biblioteca de Economía, Ediciones Orbis, Barcelona.

Sowell, T. (1999), «The Quest for Cosmic Justice», The Free Press, New York, 1999, p. 27.

Stigler, G. (1988), Memoirs of an Unregulated Economist, Basic Books Inc., Publishers, Nueva York. 